\title{
Comment on Gagala et al.: Clinical and radiological outcomes of avascular necrosis of the femoral head using autologous osteochondral transfer (mosaicplasty). Preliminary report
}

\author{
Vishal Kumar • Siddhartha Sharma • Sameer Aggarwal • \\ Vijay G. Goni \\ Received: 15 May 2013 / Accepted: 7 June 2013 / Published online: 22 June 2013 \\ (C) Springer-Verlag Berlin Heidelberg 2013
}

Dear Sir,

We read with great interest the article by Gagala et al. entitled "Clinical and radiological outcomes of avascular necrosis of the femoral head using autologous osteochondral transfer (mosaicplasty). Preliminary report" [1]. Although the authors presented their initial results with this novel technique, there are several intriguing points that we think need to be elaborated upon:

1. It is a well-known fact that the results of femoral-headpreserving procedures are dismal once collapse has occurred [Association Research Circulation Osseous (ARCO) stage III] $[2,3]$. The only study reporting the use of autologous osteochondral transfer (OATS) in postcollapse stage of osteonecrosis of the femoral head (ONFH), by Rittmeister et al. [4], confirms these findings. We would like to know the authors' rationale for choosing OATS/allograft procedure in ARCO stages IIIIV when the existing literature does not recommend such procedures for these stages. Also, the authors should clarify whether ethical clearance was sought for this procedure and informed written consent was obtained from the study participants.

2. A major objective of the study was to evaluate the radiological outcome of the OATS procedure. The authors used only X-rays for radiological evaluation. We believe that magnetic resonance imaging (MRI) would have been a better tool to identify graft incorporation and disease progression postoperatively.

V. Kumar $\cdot$ S. Sharma $\cdot$ S. Aggarwal $\cdot$ V. G. Goni

Department of Orthopaedic Surgery, Postgraduate Institute

of Medical Education and Research, Chandigarh, India

S. Sharma $(\bowtie)$

E-412, MDH, PGIMER, Sector 12, Chandigarh, India

e-mail: sids82@gmail.com
3. The authors need to elucidate their OATS surgical technique. How did they determine the exact location of the drill holes for the OATS procedure intraoperatively? How did they ensure that all necrotic bone had been removed? Was any intraoperative imaging used? The necrotic segment can be difficult to identify on naked-eye inspection when the femoral head has not collapsed, and ARCO stage IIa lesions can be difficult to identify even on image intensifiers.

4. Clarification is also needed regarding the use of morcellised allograft for ARCO stages IIC, III, and IV. How was the allograft processed and stored prior to usage? It is a wellknown fact from the literature on knee articular cartilage injuries that OATS can provide coverage only for lesions up to two centimetres. For larger lesions, allografts obtained from a fresh osteoarticular, size-matched hemicondyle are recommended [3]. Even if the defect in the femoral head (after curettage) was packed with allograft, it is hard to imagine that the authors were able to provide adequate cartilaginous coverage to the diseased femoral head, a fact that may very well have accounted for the failure of this procedure.

5. What was the authors' indication for performing a subtrochanteric derotation osteotomy in one case?

6. The natural history of ONFH, especially in the precollapse stage, is widely variable, and lesions may not progress for years. Therefore, any surgical option to defer arthroplasty should be followed up for at least five years, and ideally ten. Only seven patients in the study had a follow-up of at least five years. We therefore feel that the authors' follow-up is inadequate.

7. The authors concluded that treatment with OATS is neither better nor worse than other joint-preservation options. However, there was no control group. Furthermore, this pilot study had just 21 patients, with small number of patients in each ARCO stage, thereby precluding any meaningful statistical analysis. Comparison of these 
results with those of other studies in the literature also risks introduction of bias in the patient characteristics, risk factors for ONFH and the expertise of the operating surgeon. Therefore, we feel that such conclusions should be avoided.

To conclude, we feel that more information in the form of large, multicentre studies is needed before we can judge the usefulness of the OATS procedure for ONFH. It would also help if the authors reported the long-term results of their surviving hips.

Conflicts of interest The authors declare that they have no conflicts of interest.

\section{References}

1. Gagala J, Tarczynska M, Gaweda K (2013) Clinical and radiological outcomes of treatment of avascular necrosis of the femoral head using autologous osteochondral transfer (mosaicplasty). Preliminary report. Int Orthop May 1. doi:10.1007/s00264-013-1893-6

2. Lieberman JR, Engstrom SM, Meneghini RM, SooHoo NF (2012) Which factors influence preservation of the osteonecrotic femoral head? Clin Orthop Relat Res 470:525-534

3. Miller RH III, Azar FM (2012) Knee Injuries. In: Canale ST, Beaty JH (eds) Campbell's Operative Orthopaedics, 12th edn. Mosby, Philadelphia, pp 2183-2184

4. Rittmeister M, Hochmuth K, Kriener S, Richolt J (2005) Five-year results following autogenous osteochondral transplantation to the femoral head. Orthopade 34(320):322-326 